\title{
Biodegradable Pickering emulsions of Lipiodol for liver trans-arterial chemo-embolization
}

Frédéric Deschamps ${ }^{a, b}$, Thomas Isoardo ${ }^{c}$, Stéphanie Denis ${ }^{c}$, Nicolas Tsapis ${ }^{c}$, Lambros Tselikas $^{a, b}$, Valérie Nicolas ${ }^{d}$, Angélo Paci ${ }^{b, e}$, Elias Fattal $^{c}$, Thierry de Baere ${ }^{a, b}$, Nicolas Huang $^{c}$, Laurence Moine ${ }^{* c}$.

a Département de Radiologie Interventionnelle, Gustave Roussy, Université Paris-Saclay, 114 rue Edouard Vaillant, 94805 Villejuif, France.

${ }^{\mathrm{b}}$ Laboratoire de vectorologie et thérapeutiques anticancéreuses, UMR8203, Université ParisSaclay, 94805 Villejuif, France.

${ }^{\mathrm{c}}$ Institut Galien Paris-Sud, CNRS, Univ. Paris-Sud, Université Paris-Saclay, 92296 ChâtenayMalabry, France.

${ }^{\mathrm{d}}$ Plateforme d'imagerie cellulaire MIPSIT, SFR-UMS-IPSIT, Univ. Paris-Sud, Université Paris-Saclay, 92296Châtenay-Malabry, France.

${ }^{\mathrm{e}}$ Univ. Paris-Sud, Université Paris-Saclay, 92296 Châtenay-Malabry, France.

* Corresponding author at: CNRS, Institut Galien Paris-Sud, CNRS UMR 8612, Faculté de Pharmacie, 92296 Châtenay-Malabry, France. Tel.: +33 146835994. E-mail address: laurence.moine@u-psud.fr 


\begin{abstract}
:
Water-in-oil (W/O) Lipiodol emulsions remain the preferable choice for local delivery of chemotherapy in the treatment of hepatocellular carcinoma. However, their low stability severely hampers their efficiency. Here, remarkably stable W/O Lipiodol emulsion stabilized by biodegradable particles was developed thanks to Pickering technology. The addition of poly(lactide-co-glycolide) nanoparticles (NPs) into the aqueous-phase of the formulation led to W/O Pickering emulsion by a simple emulsification process through two connected syringes. Influence of nanoparticles concentration and water/oil ratio on emulsion stability and droplet size were studied. All formulated Pickering emulsions were W/O type, stable for at least one month and water droplets size could be tuned by controlling nanoparticle concentration from $24 \mu \mathrm{m}$ at $25 \mathrm{mg} / \mathrm{mL}$ to $69 \mu \mathrm{m}$ at $5 \mathrm{mg} / \mathrm{mL}$. The potential of these emulsions to efficiently encapsulate chemotherapy was studied through the internalization of doxorubicin (DOX) into the aqueous phase with a water/oil ratio of $1 / 3$ as recommended by the medical community. Loaded-doxorubicin was released from conventional emulsion within a few hours whereas doxorubicin from stable Pickering emulsion took up to 10 days to be completely released. In addition, in vitro cell viability evaluations performed on the components of the emulsion and the Pickering emulsion have shown no significant toxicity up to relatively high concentrations of NPs $(3 \mathrm{mg} / \mathrm{mL})$ on two different cell lines: HUVEC and HepG2.
\end{abstract}

Keywords: Pickering emulsion, Nanoparticles, Lipiodol, Doxorubicin, Hepatocarcinoma 


\section{Introduction}

Emulsions using ethiodized oil (Lipiodol) are currently used for the local delivery of chemotherapy to unresectable Hepatocellular Carcinoma (HCC) during liver Trans-Arterial Chemo-Embolization (TACE). This is a widely performed and recognized procedure owing to its selectivity for the tumor tissue over prolonged periods [1]. The advantage of using a Lipiodol emulsion for the treatment of HCC is threefold: 1) an embolic effect, enhanced in the case of a water-in-oil (W/O) emulsion [2], 2) the release of an anticancer drug dissolved in the water emulsion droplets (the emulsion acts as a transient reservoir, allowing a slow release of the chemotherapy into the tumors' supply and a prolonged exposure of the tumor cells while minimizing systemic exposures[1]), and 3) the radio-opacity of the Lipiodol, which ensures easy monitoring of the injection into the patient under X-ray guidance.

A major challenge in the formulation of W/O Lipiodol emulsions consists in the improvement of their stability. Today, their use has been limited since rapidly, the two phases of the emulsion separate in vitro as well as in vivo leading to the clearance of almost all the water soluble chemotherapeutic agent from the tumor and to high systemic exposure [2,3]. Several approaches have been attempted to improve stability. These include the use of contrast agent for preparation of chemotherapy aqueous phase, and/or the modification of technical parameters (water-in-oil vs oil-in-water, different ratios of lipiodol and drug, various emulsification techniques) [4-7]. However, no real worldwide recommendation was adopted and Lipiodol emulsions remain unstable systems. A solution, among others, is the use of stabilizers during formulation for long term stability. Emulsifiers are able to promote emulsification by decreasing the interfacial tension between the phases and to stabilize the interface between the droplets and the external phase $[3,8]$. Their adsorption at the interface leads to the formation of a protective film, which prevents droplets coalescence and phase separation, thus favoring emulsion stabilization. Although synthetic surfactants have been so far used to stabilize pharmaceutical emulsions, this kind of emulsifiers raises directly or indirectly toxicity issues $[9,10]$. In particular, for parenteral administration, cytotoxicity and haemolysis have been observed for most of them $[11,12]$.

The use of solid particles in the formulation of an emulsion allows to lower, or even to avoid, the use of synthetic surfactants, and very stable interfaces are obtained. Such emulsions stabilized by solid particles are called Pickering emulsions [13,14]. Compared with classical emulsions stabilized by surfactants, Pickering emulsions display outstanding stability, lower 
toxicity and have the ability to stabilize emulsions with large droplet size, so that a classical emulsion can be substituted by a Pickering emulsion in most applications [15]. Despite their potential, Pickering emulsions have certain characteristics less favourable for biomedical applications, one of which is the nature of the stabilizing particles. Indeed, particles are foreign bodies which may provoke a prolonged inflammatory response with potential deleterious effects. Due to their small size, particles develop a large surface of activation. Since particles are intended to be injected in the organism, they have to be biocompatible, biodegradable and their degradation products have to be rapidly eliminated to avoid any accumulation in the body. Recently, Whitby et al have reported the use of biodegradable particles as stabilizers rather than usual organic or inorganic particles such as silica, calcium carbonate, polystyrene [16]. These biodegradable particle made of poly(lactic-co-glycolic acid) (PLGA) offer the advantage of being biocompatible and rapidly eliminated from the body preventing potential inflammatory reaction by their accumulation [17-19]. However, in this study, non-pharmaceutical oils were tested and the resulting emulsions were therefore not biocompatible.

In this context, biodegradable Pickering emulsions could be considered as an attractive approach to stabilize Lipiodol emulsion. Lipiodol has already a marketing authorization as an agent for chemoembolization of HCC and is commonly injected into hepatic arteries for its ability to target liver tumors. Its combination with biodegradable and biocompatible PLGA particles will render this Lipiodol Pickering emulsion fully acceptable for injection. Nevertheless, the well-known established parameters for conventional lipiodol emulsions have to be maintained: (i) one needs to obtain a W/O emulsion for higher tumor selectivity, (ii) droplet sizes should be from 20 to $40 \mu \mathrm{m}$ since vessels diameter close to the tumor is around 40-60 $\mu \mathrm{m}$, (iii) a high viscosity is needed to increase its tumor retention, and ultimately (iv) a high loadable capacity in chemotherapeutic agent is required. Moreover, to be transposable in clinic, emulsification has to be obtained by repetitive pumping of 2 syringes (1 of chemotherapy and 1 of Lipiodol) through a 3-ways stopcock [20].

We report herein the feasibility of formulating W/O Pickering emulsion for TACE with biodegradable nanoparticles of PLGA. The impact of nanoparticle concentration and the water/oil ratio on the above mentioned parameters was studied by following the stability and the droplet size of the emulsion. The potential of this new system to load and release doxorubicin without affecting the global stability was then evaluated in-vitro. Finally, biocompatibility of each component and the whole emulsion was assessed on two cell lines: 
HUVEC human endothelial cells and HepG2, human hepatocyte carcinomas, representative of the target tissues.

\section{Materials and methods}

2.1. Materials

Poly(lactide-co-glycolide) (PLGA, 75:25) was provided by Evonik (Germany). Poly(vinyl alcohol) (PVA, molecular weight 30,000-70,000 g/mol, 87-90\% hydrolyzed), and Dtrehalose (from saccharomyces cerevisiae, $\geq 99 \%$ ) were obtained from Sigma Aldrich (France). Iron oxide nanoparticles suspension $\left(\mathrm{Fe}_{3} \mathrm{O}_{4}\right.$ with oleic acid coating, $\sim 5 \mathrm{~nm}, 25$ $\mathrm{mg} / \mathrm{mL}$ in chloroform) was provided by Ocean NanoTech (USA). Acetone was purchased from Carlo Erba (Milan, Italy). Distilled water was obtained by purification, using a water system ultrapure MilliQ Direct Type 1 (Millipore, resistivity of $18.2 \mathrm{M} \Omega . \mathrm{cm}$, France). Lipiodol® was purchased from Guerbet (France). Finally, doxorubicin hydrochloride (Adriblastin, $50 \mathrm{mg}$, lyophilized form) was provided by Pfizer (USA).

\subsection{Nanoparticles formulation}

PLGA nanoparticules (NPs) were prepared using a nanoprecipitation technique. $100 \mathrm{mg}$ of PLGA were dissolved in $10 \mathrm{~mL}$ of acetone. The PLGA solution was slowly added through a syringe pump into a $30 \mathrm{~mL}$ solution of PVA at $0.5 \% \mathrm{w} / \mathrm{w}$. This mixture was stirred for 10 hours at room temperature. Then, two successive ultracentrifugations were performed to eliminate free PVA, the pellets were collected and suspended into a solution of trehalose at $5 \% \mathrm{w} / \mathrm{w}$. For ease of handling, the NPs suspension was freeze-dried and stored at $-20^{\circ} \mathrm{C}$. The main characteristics of the NPs are summarized Table 1.

For confocal microscopy to clearly distinguish signals from doxorubicin and NPs, iron oxide containing PLGA NPs were prepared by a emulsion-evaporation process as described by Okassa et al [21].

\subsection{Nanoparticles characterization}

Size distribution and Zeta potential: The size distribution and zeta potential of NPs were measured with a Zetasizer (Nano ZS 90, Malvern Instruments, France, $633 \mathrm{~nm}$ He-Ne laser, Smoluchowski equation) operating at $25^{\circ} \mathrm{C}$ with a $173^{\circ}$ scattering angle. The NPs suspension was diluted in MilliQ water for size distribution measurements and in a $1 \mathrm{mM} \mathrm{NaCl}$ aqueous 
solution for zeta potential measurements. Three measurements were performed for each NPs formulation (Table 1).

Transmission Electron Microscopy: Transmission Electron Microscopy (TEM) was performed at I2BC (Gif-sur-Yvette, France) using a JEOL JEM-1400 operating at $80 \mathrm{kV} .5$ $\mu \mathrm{L}$ of purified suspensions of NPs $(0.5 \mathrm{mg} / \mathrm{mL})$ were deposited for $1 \mathrm{~min}$ on glow-discharged copper grids covered with formvar-carbon film. Samples were then stained two times using uranyl acetate for $10 \mathrm{~s}$. The excess solution was blotted off using a filter paper. Images were acquired using an Orius camera (Gatan Inc, USA).

Wetting properties: Wetting properties of NPs were evaluated with contact angle measurements. A NP layer was formed on a mica surface by deposition of a $45 \mu \mathrm{L}$ drop of NP suspension in water at $25 \mathrm{mg} / \mathrm{mL}$ which was dried during $15 \mathrm{~h}$ at $25^{\circ} \mathrm{C}$. Droplets of saline or of Lipiodol (volume: $5 \mu \mathrm{L}$ ) are carefully deposited on the NP layer, and the contact angle was measured at $\mathrm{t}=0$ at $20{ }^{\circ} \mathrm{C}$ with the sessile drop technique by a pendant drop tensiometer (Tracker, Teclis, France). Measurements were repeated 10 times (Table 1).

\begin{tabular}{|c|c|c|c|c|}
\hline Diameter (nm) & PdI & Zeta potential (mV) & \multicolumn{2}{|c|}{ Contact angle $\left(^{\circ}\right)$} \\
& & & Lipiodol & saline \\
\hline $170 \pm 5$ & 0.08 & $-4 \pm 1$ & $35 \pm 5$ & $61 \pm 12$ \\
\hline
\end{tabular}

Table 1. Physico-chemical properties of the PLGA nanoparticles (NPs).

\subsection{Emulsion formulation}

For the entire study, the aqueous-phase used was a suspension of PLGA NPs in physiological saline and the oil phase was Lipiodol (Guerbet, France $\left.{ }^{\circledR}\right)$. For all formulations, the Lipiodol volume was fixed at $3 \mathrm{~mL}$.

Four different NPs concentrations were tested: 5, 10, 15 and $25 \mathrm{mg} / \mathrm{mL}$ at a $1 / 3$ water/oil ratio (v/v). At a fixed NPs concentration of $15 \mathrm{mg} / \mathrm{mL}, 4$ different water/oil ratios (v/v): 1/3 ; 1/2 ; $2 / 3$ and $1 / 1$ were evaluated (Table 2). 


\begin{tabular}{|c|c|c|c|c|c|c|c|c|}
\hline \multicolumn{2}{|l|}{ Water/oil ratio } & \multicolumn{4}{|c|}{$1 / 3$} & $1 / 2$ & $2 / 3$ & $1 / 1$ \\
\hline \multirow[t]{2}{*}{ Volume (mL) } & Water & \multicolumn{4}{|c|}{1} & 1.5 & 2 & 3 \\
\hline & Oil & \multicolumn{4}{|c|}{3} & 3 & 3 & 3 \\
\hline \multicolumn{2}{|c|}{ NPs concentration $(\mathrm{mg} / \mathrm{mL})$} & 5 & 10 & 15 & 25 & \multicolumn{3}{|c|}{15} \\
\hline
\end{tabular}

Table 2. Composition of the different formulations for the preparation of emulsions with Lipiodol as the oil-phase and saline as aqueous-phase.

Emulsions were obtained by mixing through two luer lock $10 \mathrm{~mL}$ syringes coupled to a threeway stop cock. During emulsification, the water phase was slowly added at a flow rate of 1 $\mathrm{mL} / \mathrm{min}$ with a syringe pump. Sixty repetitive pumping during one minute were done to achieve emulsification [22].

For doxorubicin loaded emulsions, only the 1/3 water/oil ratio was studied at a doxorubicin concentration of $20 \mathrm{mg} / \mathrm{mL}$ and at NP concentration of $15 \mathrm{mg} / \mathrm{mL}$ was tested. This ratio was selected according to published technical recommendation for conventional transarterial chemoembolization suggesting that the volume of drug aqueous solution should be lower than the volume of Lipiodol [20]. Although, a doxorubicin dissolution step was first required in the NP suspension, they were obtained the same way as other emulsions. Conventional doxorubicin emulsion was similarly prepared without NPs and without surfactant.

\subsection{Emulsion characterization}

Stability: Emulsion stability was evaluated over 24 hours at $20^{\circ} \mathrm{C}$ by using a Turbiscan Classic MA 2000 apparatus (Formulation, Toulouse, France). The apparatus uses a near-IR light, scans the emulsion from bottom to top and a photocell calculates the intensity of the transmitted and scattered light. The freshly produced emulsion was placed properly into a tube to avoid any perturbation of the system; the tube was not removed or even touched from the apparatus until the end of the measurement. Measurements were performed every 10 minutes during the first hour, and then every hour until $24 \mathrm{~h}$.

Droplet size analysis: Droplet size measurement was performed with a particle counter, shape and size analysis apparatus (Flowcell FC200S+HR, Occhio, Belgium). Thanks to image analysis, the Flowcell apparatus measures the size and the morphology of droplets. The emulsion was first diluted 20 times in oil. Then $0.5 \mathrm{~mL}$ of the diluted emulsion was passed through a $400 \mu \mathrm{m}$ spacer to get a size range between 5 and $400 \mu \mathrm{m}$. Each sample, stored at $20^{\circ} \mathrm{C}$, was measured 5 times and at different days (D0, D7, D35). Measurements were performed on at least 500 droplets or more. Assuming a limited coalescence process 
characterized by an irreversible adsorption of all the NPs at the interface, the dependence of the droplet diameter $\mathrm{D}$ with the mass $\mathrm{m}_{\mathrm{p}}$ of NPs is given by the following equation:

$$
\frac{1}{D}=\frac{m_{p}}{4 C \rho_{p} d_{p} V_{d}}
$$

with $\mathrm{C}$ the surface coverage rate (i.e. the fraction of the droplet surface occupied by NPs), $\rho_{p}$ the particle density (for PLGA, it is $1.3 \mathrm{~g} . \mathrm{cm}^{-3}$ ), $d_{p}$ the particle diameter, $V_{d}$ the volume of the dispersed phase [23].

Microscopy: All emulsions were observed with an optical microscope (Olympus B201, Japan) at 10x optical zoom. The images were taken 1 hour, 7 days and 35 days after the emulsification. Confocal microscopy was performed using a confocal scanning laser microscope (inverted Leica TCS SP8 - gated STED, Germany) using a HC PL APO CS2 $63 \mathrm{x} / 1.40$ oil immersion objective lens. The instrument was equipped with a WLL Laser (472 $\mathrm{nm}$ excitation wavelength for doxorubicin and $633 \mathrm{~nm}$ for the reflexion mode for NPs). Red fluorescence emission from doxorubicin was collected with a 485-738 nm wide emission slits and a 592-679 $\mathrm{nm}$ wide emission slits for the reflexion signal under a sequential mode. Transmission images were acquired with the $633 \mathrm{~nm}$ laser line and a PMT-trans detector. The pinhole was set at 1.0 Airy unit. 12 bit numerical images were done with Leica SP8 LAS X software (Version 2.0.1; Leica, Germany).

Freeze-fracture electron microscopy was also performed to observe our emulsions. For each sample, three specimen carriers (gold plated copper cupules with dome shaped indentation of $1.4 \mathrm{~mm}$ central diameter) were gently filled with the corresponding formulation. The samples were then quickly frozen by plunge in liquid ethane with a Leica CPC apparatus (Leica microsystems) without cryoprotectant, and transferred in cryotubes filled with liquid nitrogen using the CPC chamber maintained at $-160^{\circ} \mathrm{C}$ with gaseous nitrogen. With the Leica VCT100 cryo preparation workstation and shuttle, samples were respectively loaded (on a holder with adapted retaining spring) and maintained frozen during transfers. With the Leica ACE 600 apparatus, samples were freeze-fractured at $-150^{\circ} \mathrm{C}$ (high vacuum around $4.10^{-6} \mathrm{mbar}$ ) and then coated by sputtering with $3 \mathrm{~nm}$ of iridium at $8.10^{-3}$ mbar and $80 \mathrm{~mA}$ in argon plasma). Finally observations were made at $-150^{\circ} \mathrm{C}$ in a Field Emission SEM Gemini 500 (Zeiss) operating at $790 \mathrm{~V}$, with a $15 \mu \mathrm{m}$ objective aperture diameter and a working distance at / around $2.2 \mathrm{~mm}$ (high vacuum around $1.10^{-6} \mathrm{mbar}$ in the observation chamber). Secondary electrons were collected with the in-lens detector. Scan speed and line or drift compensation integrations were adjusted during observations. 
Viscosity: Viscosity measurements were performed with an AR-G2 rheometer (TA Instruments, USA), equipped with a plate-plate geometry (diameter $35 \mathrm{~mm}$, gap $300 \mu \mathrm{m}$ ). Temperature was controlled with a Peltier plate at $20{ }^{\circ} \mathrm{C}$. A three-step shear rate sweep was imposed after a 2-minute equilibration time: 1) increase of the shear rate from 1 to $10000 \mathrm{~s}^{-1}$ during 2 min (upwards curve), 2) peak hold at $10000 \mathrm{~s}^{-1}$ during $30 \mathrm{~s}, 3$ ) decrease of the shear rate from $10000 \mathrm{~s}^{-1}$ during $2 \mathrm{~min}$ (downwards curve). Experiments were performed in triplicate for reproducibility.

Doxorubicin release: $0.8 \mathrm{~mL}$ of a freshly prepared doxorubicin-loaded emulsion (equivalent to an amount of $4 \mathrm{mg}$ of doxorubicin) was placed into GeBAflex dialyse tube with pores of 14 $\mathrm{kDa}$ (GeBa, Israel). The tubes were immersed into $40 \mathrm{~mL}$ of $25 \mathrm{mM}$ TRIS solution containing $150 \mathrm{mM}$ of $\mathrm{NaCl}(\mathrm{pH}=7.3)$. Then, the whole release system was placed in an orbital shaker at $37^{\circ} \mathrm{C}$ for the entire duration of the experiment. Before each aliquot withdrawing, the tubes were vortexed and the medium was entirely collected and replaced by fresh TRIS solution. For measurements, $100 \mu \mathrm{L}$ of the release medium was collected and doxorubicin concentration was determined by absorption spectrometry at a wavelength of $490 \mathrm{~nm}$ with an Elisa microwell plate reader.

Cell culture and cell viability studies: these tests were performed on two cell lines: Hep G2 (a human-derived carcinoma cell line widely used to evaluate toxic effects of drugs) and HUVEC (endothelial cells representative of the vascular endothelium). Cell culture: the human hepatocarcinoma Hep-G2 (ATCC HB-8065) and umbilical vein endothelial HUVEC (ATCC CRL-1730) cells lines were cultured in Dulbecco's Modified Eagle's Medium (DMEM) high glucose $(4.5 \mathrm{~g} / \mathrm{L})$ supplemented with $10 \%$ fetal bovine serum, $2 \mathrm{mM} \mathrm{L}$ glutamine, $100 \mathrm{U} / \mathrm{mL}$ penicillin, $100 \mathrm{mg} / \mathrm{mL}$ streptomycin and incubated in humidified atmosphere of $5 \% \mathrm{CO}_{2}$ at $37{ }^{\circ} \mathrm{C}$.

Cell proliferation assay: Cellular mitochondrial activity was evaluated after incubation with the different treatments using the 3-[4,5-dimethylthiazol-2-yl]-3,5-diphenyl tetrazolium bromide (MTT) test. Cells were seeded in growth medium and allowed to adhere for $24 \mathrm{~h}$. The initial cell densities and incubation times were determined to allow the cells to remain in exponential growth.

For Doxorubicin and NPs: 5000 cells were seeded in $100 \mu \mathrm{L}$ of growth medium in 96-well plates (TPP), and allowed to adhere for $24 \mathrm{~h}$. Cells were then treated with $100 \mu \mathrm{L}$ of 2 times concentrated test compounds at a series of concentrations and incubated at $37^{\circ} \mathrm{C}$ for $48 \mathrm{~h}$. 
For Lipiodol and emulsions: due to their viscosity, a homogenous mother solution couldn't be prepared so each well of a 12 wells plate was treated individually in triplicate. 54000 cells were seeded in $2000 \mu \mathrm{L}$ of growth medium in 12-well plates (TPP), and allowed to adhere for $24 \mathrm{~h}$. Wells were then treated with the adequate volume of the product and medium was added to have a final volume of $2 \mathrm{ml}$. Plates were then incubated at $37^{\circ} \mathrm{C}$ for $48 \mathrm{~h}$.

At the end of the incubation period, MTT (Sigma-Aldrich; $5 \mathrm{mg} \cdot \mathrm{mL}^{-1}$ solution in phosphate buffered saline; $20 \mu \mathrm{L} / 200 \mu \mathrm{l}$ ) was added to each well. After 1 to $4 \mathrm{~h}$ of incubation at $37^{\circ} \mathrm{C}$, the culture medium was removed and replaced by DMSO $(200 \mu \mathrm{L} / 1 \mathrm{ml})$ in order to lyse the cells and dissolve the formazan crystals. After $5 \mathrm{~min}$ of stirring, the absorbance of the solubilized dye was measured spectrophotometrically with a microplate reader (LAB System Original Multiscan MS) at $570 \mathrm{~nm}$. The percentage of viable cells for each treatment was calculated from the ratio of the absorbance of the well containing the treated cells vs. the average absorbance of the control wells (i.e. untreated cells). All experiments were set up in triplicate to determine means and standard deviations.

\subsection{Statistical analysis}

All results were mentioned as mean values \pm standard deviation (SD).

\section{Results}

\subsection{Nanoparticles preparation and characterization}

PLGA NPs were formulated by a simple versatile nanoprecipitation process. Transmission Electron Microscopy showed relatively homogeneous particle sizes with spherical and smooth surfaces (Figure 1). The mean hydrodynamic particle diameter was found around $170 \mathrm{~nm}$ with a narrow polydispersity consistent with TEM observations. A slightly negative zeta potential was measured which is common for PVA coated NPs [24].

According to contact angle analyses (Table 1), NPs were found amphiphilic, and more hydrophobic than hydrophilic, and consequently might favor the formation of W/O emulsion. 


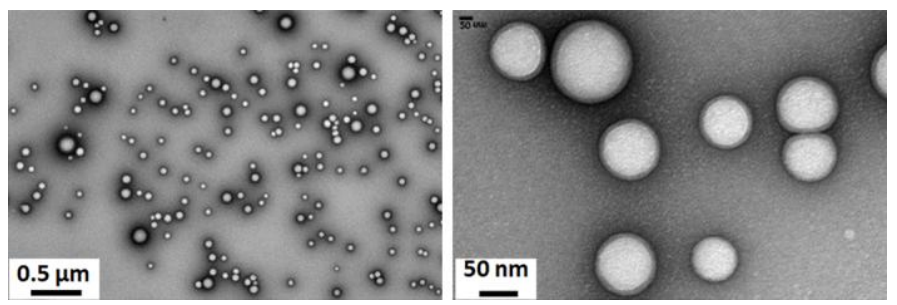

Figure 1. Transmission Electron Microscopy images of dried PLGA NPs.

\subsection{Emulsion formulation}

\subsubsection{Influence of NP concentration}

The importance of solid particles concentration during formulation of Pickering emulsions was highlighted in previous reports $[15,16]$. In particular, reducing the amount of particles in the formulation led to the formation of very large droplets since only a small interfacial area can be stabilized. The influence of NP concentration on the Lipiodol emulsion formulation was therefore evaluated through the stability and droplet size of the resulting emulsions.

Four different concentrations of NPs were tested: $5,10,15$ and $25 \mathrm{mg} / \mathrm{mL}$ at a water/oil ratio of $1 / 3$. All the emulsions were successfully prepared and they were all W/O emulsions. Microscopic aspects of the different emulsions are shown in Figure S1 in supplementary material. In all cases, aqueous droplets are relatively well dispersed in the continuous Lipiodol phase. A darker interface between the aqueous core of the droplets and Lipiodol could be distinguished. One could also observe that the droplets size is strongly influenced by the NP concentration used for each formulation. Finally, droplets were generally slightly less spherical when NP concentrations increased.

To characterize the darker interface, confocal microscopy was performed using iron oxide containing PLGA NPs for their visualization (Figure 2a). The same global aspect was found compared to optical microscopy but the outlines of the droplets were highlighted by the presence of the NPs adsorbed at the interface. This presence was also confirmed in freezefracture electron microscopy (Figure 2b). With this technique, a dense NP monolayer adsorbed at the droplet interface could be observed. 
a)

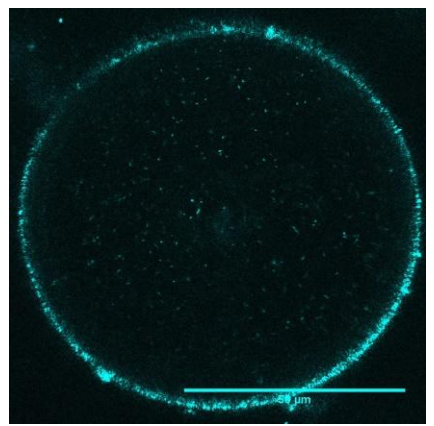

b)

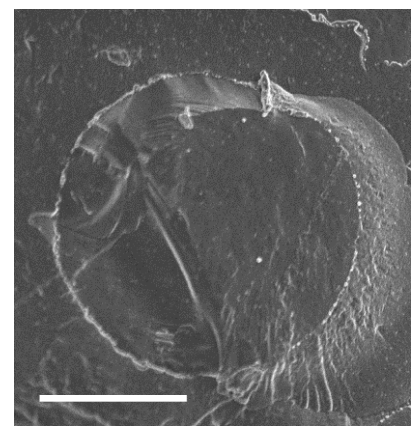

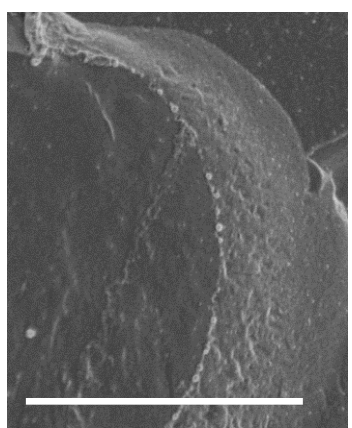

Figure 2. Images of the W/O Lipiodol Pickering emulsions at $15 \mathrm{mg} / \mathrm{mL}$ of PLGA NPs with a 1/3 water/oil ratio in: a) confocal microscopy (scale bar $=50 \mu \mathrm{m}$ ); b) freeze-fracture electron microscopy (scale bar $=5 \mu \mathrm{m})$.

Immediately after formulation, evolution of the emulsion stability was followed by static light scattering (Turbiscan ${ }^{\circledR}$ ) during $24 \mathrm{~h}$ (curves are displayed in supplementary material Figure S2). As shown in Table 3 and Figure S3, whatever the NP concentration, creaming was rapidly observed after formulation. Creaming is the phenomenon of less dense droplets (water droplets in our case) rising to the top to form a dense emulsion layer, with no evolution of the drop size. This is a reversible phenomenon which generally occurs when droplet size is relatively large. There is only a gradual transition in the water droplet concentration with no evolution of the droplet size. A gentle manual agitation is sufficient to re-homogenize the emulsion with a uniform distribution of the droplets [25]. The duration and the relative height of the creaming both increased with the NPs concentration. For the 10,15 and $25 \mathrm{mg} / \mathrm{mL}$ formulations, phase separation (in our case, droplets destabilization leading to a thin aqueous layer on top of the emulsion) was not observed during the time of analysis. Concerning the 5 $\mathrm{mg} / \mathrm{mL}$ emulsion, phase separation started immediately after emulsification and was stabilized after $2 \mathrm{~h}$, causing an upper aqueous layer of a relative height of $3 \pm 0.3 \%$ compared to the total height of the solution. 


\begin{tabular}{|c|c|c|c|c|}
\cline { 2 - 4 } \multicolumn{1}{c|}{} & $5 \mathrm{mg} / \mathrm{mL}$ & $10 \mathrm{mg} / \mathrm{mL}$ & $15 \mathrm{mg} / \mathrm{mL}$ & $25 \mathrm{mg} / \mathrm{mL}$ \\
\hline Creaming duration (h) $^{c}$ & $2.0 \pm 0.2$ & $4.5 \pm 0.5$ & $5.5 \pm 0.5$ & $9.0 \pm 0.1$ \\
\hline Relative height of creaming $^{\mathrm{a}}(\%)$ & $48 \pm 2$ & $62 \pm 1$ & $73 \pm 1$ & $90 \pm 1$ \\
\hline Relative height of phase separation $^{\mathrm{b}}(\%)$ & $3 \pm 0.3$ & 0 & 0 & 0 \\
\hline
\end{tabular}

Table 3. Emulsion stability of W/O Lipiodol Pickering emulsions with different NP concentrations at $24 \mathrm{~h}$, at a water/oil ratio of $1 / 3$. ${ }^{\text {a) }}$ Height of the emulsion layer / total height,

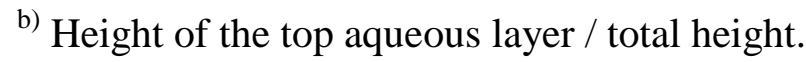

Average droplet size after emulsification (D0) decreased as the NP concentration increased, from $69 \mu \mathrm{m}$ at $5 \mathrm{mg} / \mathrm{mL}$ to $24 \mu \mathrm{m}$ at $25 \mathrm{mg} / \mathrm{mL}$ (Figure $3 \mathrm{a}$ ). At $5 \mathrm{mg} / \mathrm{mL}$, the diameter gradually increased with time (from $58 \mu \mathrm{m}$ at D0 to $120 \mu \mathrm{m}$ at D35) with large standard deviations, whereas the droplet sizes remained stable during 35 days at higher NPs concentrations $(10,15$ and $20 \mathrm{mg} / \mathrm{mL})$.

a)

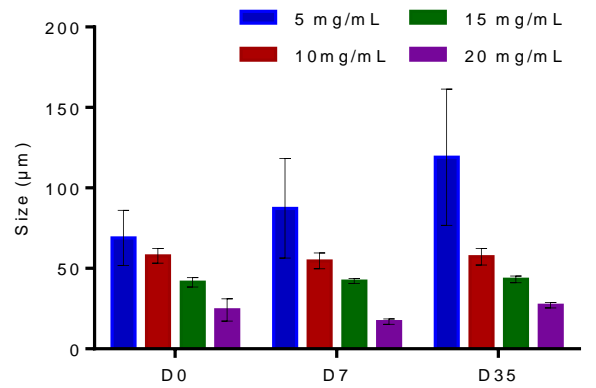

b)

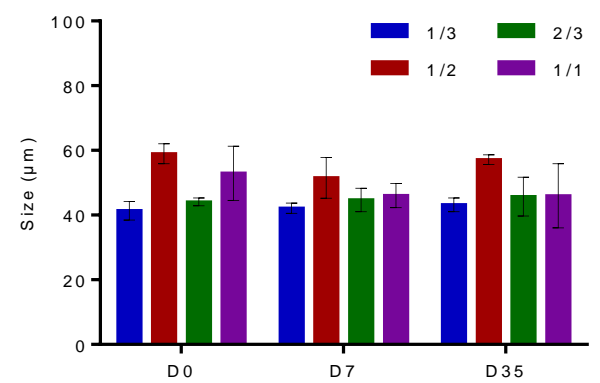

Figure 3. Droplet size of the Lipiodol Pickering emulsions a) at water/oil ratio of $1 / 3$ with different NPs concentrations and b) at a fixed NPs concentration of $15 \mathrm{mg} / \mathrm{mL}$ with different water/oil ratios at D0, D7 and D35.

At D35, a linear relationship between the inverse of the droplet size and the NP concentration could be established $\left(r^{2}=0.98\right)$ (Figure 4$)$. 


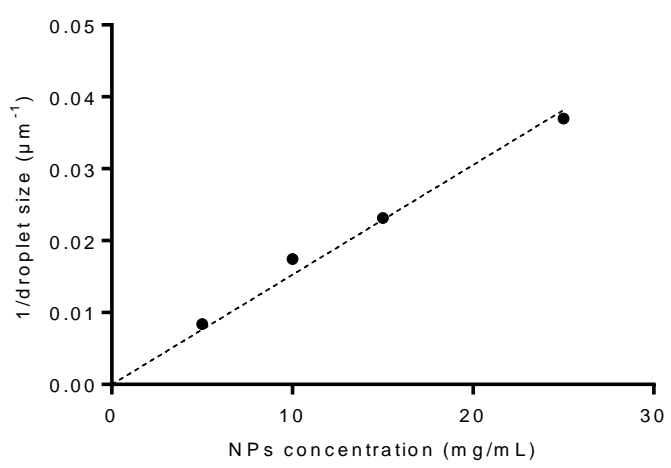

Figure 4. Inverse droplet size as a function of the NPs concentration at D35.

\subsubsection{Influence of water/oil ratio}

In addition to studying the impact of NP concentration, the effects of changing the water/oil ratio while keeping the rest of formulation constant was also investigated. The concentration of $15 \mathrm{mg} / \mathrm{mL}$ was selected to perform this study, as for this concentration, droplet size is more suitable for TACE [20].

All the emulsions were successfully prepared with the desired W/O type. Microscopic images show similar aspects for emulsions 1/3,1/2 and 2/3 whereas emulsion 1/1 exhibits more heterogeneous morphologies (supplementary material Figure S4). Indeed, presence of some large droplets could be observed. Inside these larger droplets, smaller droplets could be distinguished. This observation was confirmed by confocal microscopy (supplementary material Figure S5).

All emulsions were stable without phase separation for 24 hours according analysis by static light scattering (supplementary material Figure S6).

For emulsions $1 / 3$ and $1 / 2$, a creaming was rapidly observed after their preparation which stabilized after 5-6h (Table 4 and Figure S7). The relative creaming height was higher for emulsion $1 / 2$ than for emulsion $1 / 3$ (91\% and $73 \%$ respectively). For the two other emulsions, $2 / 3$ and $1 / 1$, the creaming phase filled the entire system at least during the first 24 hours. 


\begin{tabular}{|c|c|c|c|c|}
\cline { 2 - 4 } \multicolumn{1}{c|}{} & $1 / 3$ & $1 / 2$ & $2 / 3$ & $1 / 1$ \\
\hline Creaming phase duration (h) & $5.5 \pm 0.5$ & $6.0 \pm 0.5$ & - & - \\
\hline Relative height of creaming phase (\%) & $73 \pm 1$ & $91 \pm 1$ & 100 & 100 \\
\hline
\end{tabular}

Table 4. Emulsion stability of W/O Lipiodol Pickering emulsions at a NPs concentration of $15 \mathrm{mg} / \mathrm{mL}$ with different water/oil ratios at $24 \mathrm{~h}$.

The change of the water/oil ratio did not lead to a clear effect on the average droplet size of the emulsions (Figure 3b). Despite a significantly higher droplet size for the emulsion with a water/oil ratio of $1 / 2$, they were all in the $40-60 \mu \mathrm{m}$ size range. After 7 and 35 days, no significant change was observed.

\subsubsection{Viscosity of the emulsions}

Another important characteristic of Lipiodol Pickering emulsions is their viscosity which is affecting their injectability through microcatheter and its ability to homogeneously distribute to the hepatic arteries [26]. The effect of water/oil ratio on the flow behavior of the Pickering emulsion was investigated at a fixed NP concentration of $15 \mathrm{mg} / \mathrm{mL}$ where the droplet size is optimum for the chemo-embolization (Figure 5). All emulsions behaved as a non-Newtonian shear-thinning fluid as the viscosity decreased with increasing shear rate whereas Lipiodol had a Newtonian behavior (Figure 5a). This typical shear-thinning behavior is attributed to the progressive ordering of the emulsion droplets at increasing shear rates [27]. At high shear rates, viscosities decreased down to the viscosity of Lipiodol, which could allow the injection of the emulsions with a reasonable force. Moreover, emulsions exhibited a hysteresis loop in the viscosity curves with upwards curves viscosities higher than downwards curves viscosities (Figure 5b), which was more remarkable as the amount of water droplets increased. This indicates that these emulsions demonstrated a thixotropic behavior depending on the water/oil ratio (the more droplets in the emulsion, the stronger the thixotropic behavior). 

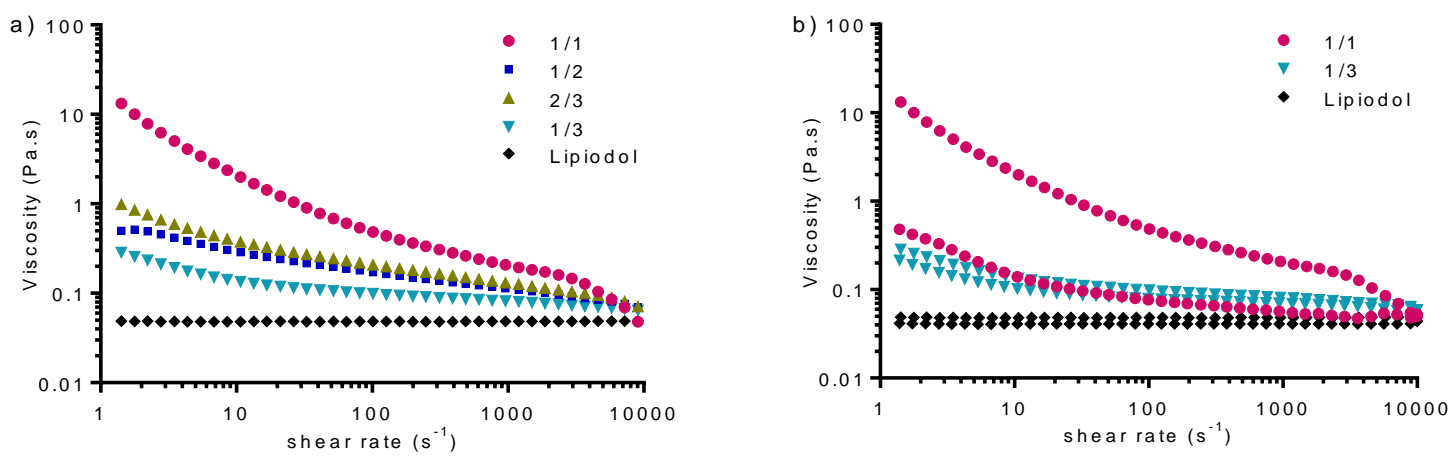

Figure 5. a) Viscosity vs shear rate (upwards curve) of Lipiodol Pickering emulsions at a fixed NP concentration of $15 \mathrm{mg} / \mathrm{mL}$ and at water/oil ratios of 1/3,1/2, 2/3, and 1/1 ; b) Viscosity vs shear rate (upwards and downwards curves); for emulsions, only ratios 1/3 and 1/1 are plotted for clarity. Upwards curves viscosities are higher than downwards curves viscosities.

The presence of water droplets also significantly increased the viscosity compared to the viscosity of Lipiodol (Figure 5). Indeed, it can be seen from Figure 6 that the relative viscosity $\eta_{r}=\eta / \eta_{s}$ (with $\eta$ the viscosity of the emulsion and $\eta_{s}$ the viscosity of the solvent, here Lipiodol) increased sharply as a function of the water volume fraction $\varphi$. The obtained data are well described by the Quemada Model for hard spheres [28]: $\eta_{r}=\left(1-\varphi / \varphi_{m}\right)^{-2}$, with $\varphi_{m}$ the maximum volume fraction of the water droplets. Data fitting led to $\varphi_{m}=0.59$ $(\mathrm{R}=0.993)$, which is close to the random close packing of monodisperse hard spheres $(\approx 0.64)$ [27]. Thus, water droplets interacted as hard spheres during flow, with no droplet break-up or deformation and no formation of droplet aggregates (which could for example trap the solvent and virtually increase the droplet volume fraction). 


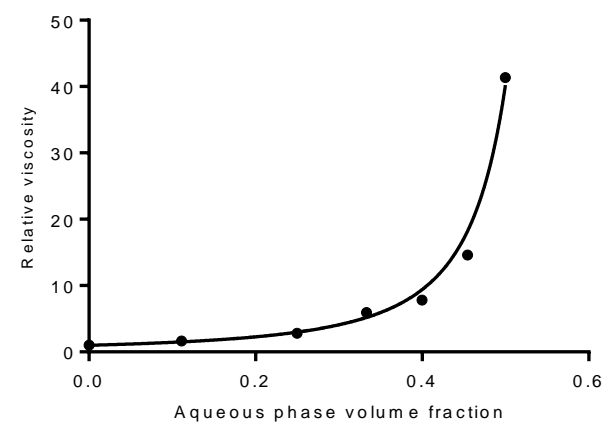

Figure 6. Relative viscosity vs water volume fraction for a shear rate of $5 \mathrm{~s}^{-1}$. Fit curve followed the Quemada model for dispersed systems.

\subsection{Doxorubicin loaded emulsion}

Liver TACE typically involves injection of water-soluble chemotherapies such as doxorubicin, epirubicin, mitomycin and cisplatin emulsified with Lipiodol. In particular, doxorubicin hydrochloride is recognized as the standard first-line drug for the treatment of HCC [20].

To fulfil all the requirements for a suitable Lipiodol Pickering emulsion as described in the introduction, doxorubicin-loaded emulsion was prepared from an aqueous solution of doxorubicin at $20 \mathrm{mg} / \mathrm{mL}$ using a water/oil ratio of $1 / 3$ and a NP concentration of $15 \mathrm{mg} / \mathrm{mL}$. After pumping, a dark red emulsion of the desired W/O type was obtained. No specific difference could be observed with the unloaded emulsion prepared with saline by optical microscopy (Figure 7). In confocal images, the same type of morphology was also observed but with additional droplet red cores which correspond to the presence of doxorubicin inside the aqueous dispersed phase. 
(A)

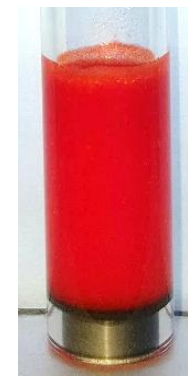

D0
(B)

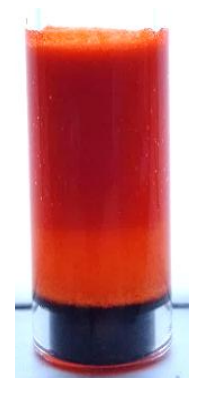

D1

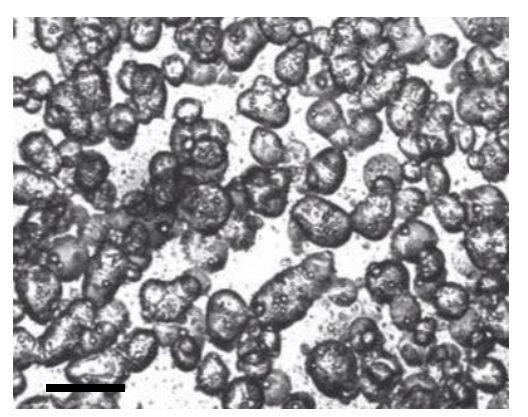

(C)

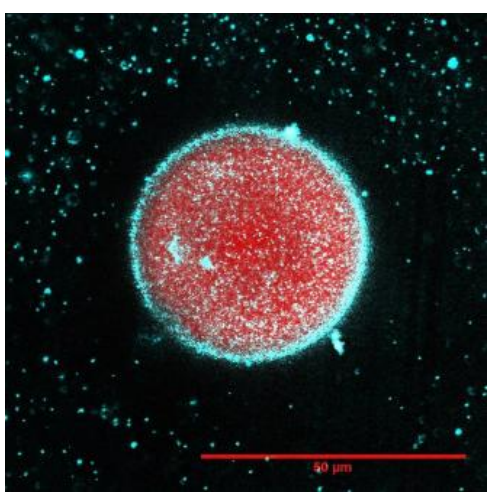

Figure 7. Doxorubicin loaded emulsion (water/oil ratio $=1 / 3,[\mathrm{NPs}]=15 \mathrm{mg} / \mathrm{mL}$, [doxorubicin] $=20 \mathrm{mg} / \mathrm{mL}$ ), (A) Images at D0 and D1, (B) Optical microscopy at D0 (scale bar $=100 \mu \mathrm{m}$ ) and (C) Confocal microscopy at D0, NPs are labelled in blue and doxorubicin in red (scale bar $=50 \mu \mathrm{m})$.

The stability of the loaded emulsion shows similar behaviour than the unloaded emulsion. Rapidly, a creaming phase appeared but no phase separation was observed (supplementary material Figure S8).

The doxorubicin release profile of Pickering emulsion and the control solutions are shown in Figure 8. After $4 \mathrm{~h}$ of incubation in TRIS, only 5\% was released from the Pickering emulsion compared to $60 \%$ for the conventional emulsion. After $24 \mathrm{~h}$, the released was complete for the Lipiodol emulsion while around 30\% was measured for the Pickering emulsion. The release of doxorubicin from Pickering emulsion was significantly sustained compared to the one from conventional Lipiodol emulsion. 


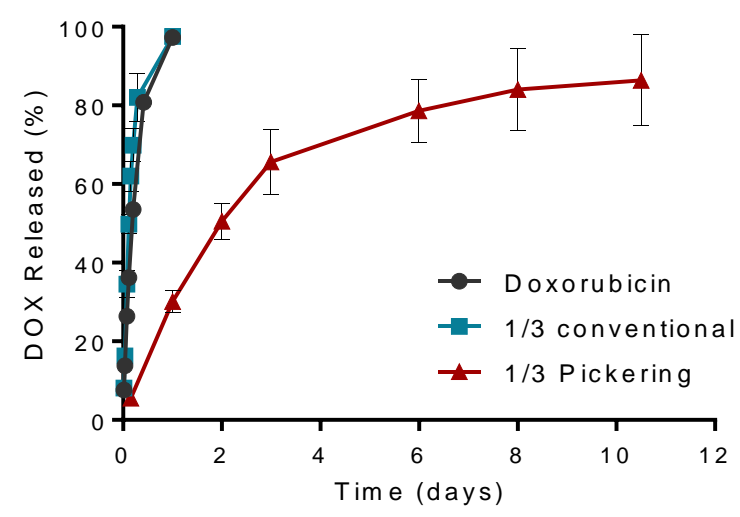

Figure 8. Doxorubicin release profiles from conventional Lipiodol emulsion (water/oil ratio = $1 / 3,[$ doxorubicin $]=20 \mathrm{mg} / \mathrm{mL}$ and Pickering emulsion $($ water/oil ratio $=1 / 3,[\mathrm{NPs}]=15$ $\mathrm{mg} / \mathrm{mL}$, [doxorubicin] $=20 \mathrm{mg} / \mathrm{mL}$ ).

\subsection{In vitro cytotoxicity studies}

\subsubsection{PLGA nanoparticles}

At low and moderate concentrations $(<3 \mathrm{mg} / \mathrm{mL})$, cell viability slightly decreases as NPs concentration increases for both cell types but remains above 65-70 \% (Figure 9). At higher concentration $(>3 \mathrm{mg} / \mathrm{mL}$ ), cellular viability decreased down to $40 \%$ at $10 \mathrm{mg} / \mathrm{mL}$ on HUVEC and this effect is more pronounced on Hep G2 with a decrease down to $20 \%$.

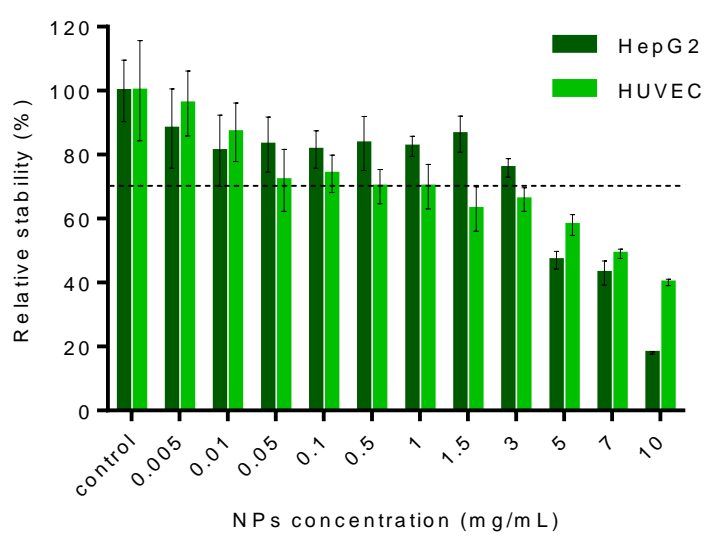

Figure 9. Viability assays on Hep G2 and HUVEC cell lines after 48h incubation with PLGA NPs. Results are presented as mean \pm SD $(n=3)$.

\subsubsection{Lipiodol and emulsions}


The impact of exposure to pure Lipiodol and Pickering emulsions loaded or not with doxorubicin, on cell viability was monitored at different dilutions ranging from 1/1000 to 1/10 on HUVEC and Hep G2 cell lines (Figure 10). The individual components of the empty emulsion induced only a moderate decrease of cell viability whatever the dilution and the cell lines. Besides, free doxorubicin at the same dilution led to a decrease of $90 \%$ of the cell viability as expected for these concentrations above $\mathrm{IC}_{50}(0.5 \mu \mathrm{M}$ for Hep $\mathrm{G} 2$ and $4 \mu \mathrm{M}$ for HUVEC, data in supplementary material). Regarding the in vitro cytotoxicity of the unloaded emulsion, only a minor reduction of the cell viability was noticed on HUVEC similar to the one of the individual components. In case of Hep G2, after $48 \mathrm{~h}$ incubation, cell viability is slightly lower than the one observed on HUVEC, but remains above $70 \%$ for quite low dilutions as $1 / 50\left(\mathrm{C}_{\mathrm{NPs}}=0.75 \mathrm{mg} / \mathrm{mL}\right)$. For doxorubicin-loaded emulsion, similar cytotoxic effect to free doxorubicin was observed on the two cell lines. After $48 \mathrm{~h}$ incubation, the loaded-emulsion should have released almost 50\% of the internalized doxorubicin corresponding for the highest dilution (1/1000) to a doxorubicin concentration of $40 \mu \mathrm{M}$ which is far above the $\mathrm{IC}_{50}$ of doxorubicin.
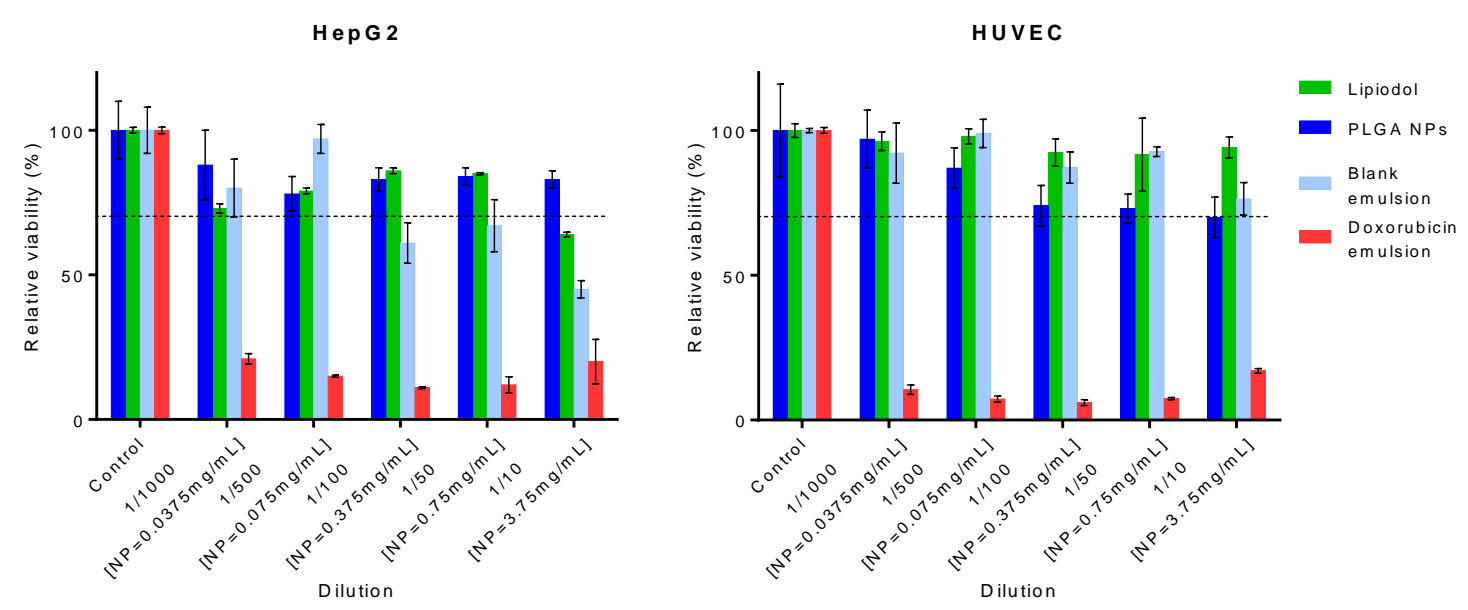

Figure 10. Viability assays on Hep G2 and HUVEC cell lines after $48 \mathrm{~h}$ incubation with PLGA NPs (nanoparticle-equivalent concentrations in brackets), Lipiodol, blank Pickering emulsion and doxorubicin loaded Pickering emulsion at different dilution times. For all dilution, cell viability of equivalent doxorubicin concentration in solution is always below 20 $\%$ for both cell lines (data are displayed in supplementary material Figure S9). Results are presented as mean $\pm \operatorname{SD}(\mathrm{n}=3)$. 


\section{Discussion}

Solid nanoparticles are noticeable alternatives to synthetic surfactants for the stabilization of emulsions. Thanks to their strong adsorption at the interface of two immiscible liquids, highly stable emulsions also called Pickering emulsions can be obtained. In many Pickering emulsion studies, the oils have been relatively simple in structure (hydrocarbon or silicone) and generally present lower density than water. These studies conclude that oil ratio and particle wetting properties have a strong influence on the emulsion type $[29,30]$.

Lipiodol emulsion has been used for TACE procedure for over 30 years for its preferential tropism for the tumor compared to surrounding tissues [31]. Many studies have attempted to stabilize this emulsion but with limited success. This failure can be attributed to the chemical nature of the oil. Lipiodol is an oily mixture of iodinated poppy-seed fatty acid esters. Due to the presence of numerous iodine atoms, the density of Lipiodol is $1.28 \mathrm{~g} / \mathrm{cm}^{3}$. The high iodine content combined to the high density contributes largely to difficulties encountered until now for the stabilisation of Lipiodol based emulsions due to physico-chemical differences with commonly used oils.

Our results have demonstrated a new strategy to stabilize Lipiodol emulsion. The addition of PLGA NPs into the aqueous-phase of the formulation has led to inverse W/O Pickering emulsion by a simple emulsification process through two connected syringes. The resulting emulsion displayed remarkable stability against coalescence and maintained their morphology over several weeks thanks to the NPs which efficiently stabilize the droplets interface against destabilization. Their injectability could be facilitated by their shear thinning behavior at elevated shear rates as applied in microcatheter during injection. Moreover, the emulsion can be formulated in presence of high concentration of doxorubicin without affecting its characteristics.

We selected PLGA NPs as emulsifier since they are used in many biomedical applications because of their biodegradability and biocompatibility. Few publications address emulsion stabilization with biodegradable NPs. Laredj-Bourezg et al demonstrated that particles of poly(caprolactone)-block-poly(ethylene glycol) [32] and of poly(lactic acid)-blockpoly(ethylene oxide) [33] were able to stabilize $\mathrm{O} / \mathrm{W}$ emulsions using a triglyceride oil for topic applications. Earlier, Whitby et al have reported the use of PLGA NPs as emulsifiers [16]. They showed that these particles preferentially stabilise oil-in-water emulsions from a wide range of oils at equal volume of oil and aqueous-phase, and did not observe a phase 
inversion at higher oil ratio. Qi et al obtained stable O/W emulsion using PLGA NPs and octanol as the oil phase for a large range of oil ratio, and also did not observe a phase inversion at high oil ratio (emulsions were unstable instead); on the contrary, the use of hexadecane led to an unstable emulsion [34]. In both studies, PVA was used to prepare the NPs, and PVA was adsorbed on the surface of the NPs, resulting in less hydrophobic NPs and promoting O/W emulsions. Despite this trend, in our study, highly stable Lipiodol emulsions were achieved over a large range of oil volume fraction and NPs concentrations. PVA was also used during the preparation of the NPs, but NPs hydrophobicity led to W/O emulsions for water/oil ratio of $1 / 1$ or below, as desired for TACE application. As highlighted by microscopy analyses, the formed droplets were surrounded by PLGA NPs indicating that these were able to adsorb at the water/Lipiodol interface.

At a NP concentration of $5 \mathrm{mg} / \mathrm{mL}$, the average droplet size increased over time, indicating a coalescence phenomenon: the amount of NPs was not enough to cover efficiently all the droplets and to prevent them to coalesce and to form larger droplets. On the contrary, at 10, 15 and $25 \mathrm{mg} / \mathrm{mL}$, the emulsions were stable already and the droplet size did not evolve significantly from D0 to D35, because NPs cover efficiently droplet surface and prevent coalescence. Stability of an emulsion stabilized by NPs is mainly governed by a steric mechanism, with NPs forming a shell around the droplets, and more NPs allow to stabilize a larger interface, and consequently to obtain more and smaller droplets. Once the interfacial area is completely covered by NPs, droplets coalescence stops: this is the phenomenon of limited coalescence $[23,35]$. This process was confirmed at D35 by the linear relationship between the inverse of the droplet size and the NP concentration (equation (1) and Figure 4), indicating that the droplets reached their final size, even for NP concentration of $5 \mathrm{mg} / \mathrm{mL}$. For all studied concentrations, the surface coverage rate value was comprised between 0.8 and 0.9, corresponding to a very dense NPs monolayer packing at the water/oil interface [23], which seemed consistent with confocal and electron microscopy pictures (Figures 2 and 7).

In 1995, de Baere et al have demonstrated a higher efficacy of Lipiodol emulsions when the aqueous droplets have a similar size as the tumor feeding arteries i.e. around $40 \mu \mathrm{m}$ [26]. Therefore, the NPs concentration was set at $15 \mathrm{mg} / \mathrm{ml}$ corresponding to an average droplet size around $40 \mu \mathrm{m}$ for the preparation of stable Lipiodol Pickering emulsions.

There is a strong interest to vary the water/oil ratio for chemoembolization. Today, there is a consensus for the preparation of Lipiodol-drug emulsion with a water/oil ratio of $1 / 3$ to form relatively stable emulsion [5]. But, for many drugs such as oxaliplatin, the amount of drug is 
limited by the concentration of the commercially available vial. Therefore, an increase of the aqueous-phase ratio may allow to incorporate more chemotherapies for TACE procedure. In this work, the Lipiodol Pickering emulsions were stable over a large range of aqueous volume fraction to an equal volume of oil, providing sufficient choice for the surgeon to load desired concentrations of chemotherapies.

Doxorubicin hydrochloride is an antineoplastic agent widely used in chemoembolization for palliative treatment of HCC. Although doxorubicin is a potent antitumor agent, its clinical utility is hampered by its toxicities such as cardiac damage and myelosuppression. Its delivery through a W/O Lipiodol emulsion system was expected to reduce/limit this toxicity. Despite a recent study that has shown a significant decrease of the plasmatic concentration using a Lipiodol emulsion prepared with contrast medium rather than saline, this system still fails to show a sustained release and lowering the systemic exposure [36].

Efficient encapsulation of the anticancer drug doxorubicin into the Pickering-emulsion was simply performed by mixing the doxorubicin aqueous-phase containing NPs with Lipiodol. In spite a high doxorubicin concentration $(20 \mathrm{mg} / \mathrm{mL})$, the resulting emulsion was stable and no specific difference could be depicted with the unloaded emulsion of the same composition. The hydrophilic drug is retained in the internal phase due to its water solubility. The addition of PLGA NPs not only improved the stability of the emulsion but also improved considerably doxorubicin release. This sustained release might arise from the formation of a strong shell created by the NPs layer surrounding aqueous droplets which slow down the diffusion process. Further experiments are required to elucidate the release mechanism and investigate the influence of W/O ratios on the drug release. However, although NPs degradation was not specifically studied, it is unlikely the reason for the diffusion slowdown. Indeed, the emulsion was stable over a 35 day-period, with constant droplet size. In case of degradation of NPs during this period, droplet size is expected to change, and probably to increase, due to the degradation of the NP shell surrounding the droplet. This would promote destabilization phenomena such as coalescence which was not observed. Moreover, the doxorubicin release for the Pickering emulsion was very progressive over more than 10 days at $37^{\circ} \mathrm{C}$ (Figure 8), which may indicate either no degradation or a slow NP degradation under these experimental conditions. 
Despite the increasing interest in Pickering technology over the last decade, very few studies concerning their cytotoxicity have been reported. As desired for biomedical applications, the Lipiodol Pickering emulsion should not release toxic sub-products or produce adverse effects after its injection. Therefore, in vitro tests were performed with all the components of the emulsion and with the entire emulsion on endothelial cells and liver cancer cells. The results of this investigation showed that the PLGA NPs do not induce specific toxicity until relatively high concentration of $3 \mathrm{mg} / \mathrm{mL}$. This is consistent with previous findings of Grabowski et al that shown a similar cytotoxicity threshold on macrophages for this type of NPs [18]. However, a NPs concentration of $3.75 \mathrm{mg} / \mathrm{mL}$ is required to stabilize a $1 / 3$ emulsion. In static cell culture environment, this NPs concentration led to a decrease of roughly $40 \%$ of the cell viability. It is of interest to note that transposition in much more dynamic physiological environment in which NPs concentration in contact with cells will be much lower and also subject to constant liquid replacement, would minimize the potential cytotoxicity. Nevertheless, additional in vivo studies will be required to evaluate the biocompatibility and the biodegradability of the NPs and the unloaded emulsion. To gain more information about the potential adverse effects of the NPs, genotoxicity assays will also be performed.

In the investigated dilution range, both doxorubicin in solution and doxorubicin loaded emulsion had similar cytotoxic effect on cells. Therefore, incorporation of doxorubicin in the emulsion did not modify its effect against tumor cells.

\section{Conclusions}

In this work, we successfully developed a Lipiodol emulsion which remains stable over weeks. This emulsion answered the main requirements to allow optimum tumor uptake by TACE procedure: (1) stable at least 24h, (2) water-in-oil emulsion for higher tumor selectivity, (3) droplet sizes around $40 \mu \mathrm{m}$ since vessels close to the tumor are between 40-60 $\mu \mathrm{m}$ (4) loadable with a chemotherapeutic agent and ultimately (5) a sustained release of the chemotherapy to extend the tumor exposure. Results are very encouraging and this combination allows the assumption that the Lipiodol Pickering emulsion will lead to better efficacy and safety than the conventional emulsion. Moreover, these stable Lipiodol Pickering emulsions over a large range of aqueous volume fraction to an equal volume of oil, provide sufficient choice for the surgeon to load desired concentrations of chemotherapies. Future work will consist in performing in vivo studies to confirm the potential of this system to be used as new agent for TACE procedure. 


\section{Acknowledgements}

The present work has benefited from the core facilities of Imagerie- Gif, (http://www.i2bc.paris- saclay.fr), member of IBiSA (http://www.ibisa.net), supported by "France- BioImaging" (ANR- 10- INBS- 04- 01), and the Labex "Saclay Plant Science" (ANR-11- IDEX- 0003-02). We thank Alexis Canette and Michaël Trichet (Electron Microscopy Facility, Sorbonne-Université, Institut de Biologie Paris-Seine FR3631) for electron microscopy analyses. N.Huang acknowledges Agence Nationale de la Recherche (ANR) for its support through a Young Researchers grant (ANR-16-CE09-0003).

\section{References}

[1] European Association For The Study Of The Liver, European Organisation For Research And Treatment Of Cancer, EASL-EORTC Clinical Practice Guidelines: Management of hepatocellular carcinoma, J. Hepatol. 56 (2012) 908-943. doi:10.1016/j.jhep.2011.12.001.

[2] H. Demachi, O. Matsui, H. Abo, H. Tatsu, Simulation model based on non-newtonian fluid mechanics applied to the evaluation of the embolic effect of emulsions of iodized oil and anticancer drug, Cardiovasc. Intervent. Radiol. 23 (2000) 285-290. doi:10.1007/s002700010070.

[3] R.C. Gaba, S. Baumgarten, B.O. Omene, R.B. van Breemen, K.D. Garcia, A.C. Larson, R.A. Omary, Ethiodized Oil Uptake Does Not Predict Doxorubicin Drug Delivery after Chemoembolization in VX2 Liver Tumors, J. Vasc. Interv. Radiol. 23 (2012) 265-273. doi:10.1016/j.jvir.2011.10.022.

[4] J.W. Choi, H.J. Cho, J.H. Park, S.Y. Baek, J.W. Chung, D.D. Kim, H.C.K. Kim, Comparison of drug release and pharmacokinetics after transarterial chemoembolization using diverse Lipiodol emulsions and drug-eluting beads, PLoS One. 9 (2014) 1-14. doi:10.1371/journal.pone.0115898.

[5] T. Masada, T. Tanaka, H. Nishiofuku, Y. Fukuoka, T. Sato, S. Tatsumoto, N. Marugami, K. Kichikawa, Techniques to Form a Suitable Lipiodol-Epirubicin Emulsion by Using 3-Way Stopcock Methods in Transarterial Chemoembolization for Liver Tumor, J. Vasc. Interv. Radiol. 28 (2017) 1461-1466. doi:10.1016/j.jvir.2017.03.032.

[6] T. Hino, Y. Kawashima, S. Shimabayashi, Basic study for stabilization of w / o / w emulsion and its application to transcatheter arterial embolization therapy, Adv. Drug Deliv. Rev. 45 (2000) 27-45.

[7] S.W. Yi, Y.H. Kim, I.C. Kwon, J.W. Chung, J.H. Park, Y.W. Choi, S.Y. Jeong, Stable lipiodolized emulsions for hepatoma targeting and treatment by transcatheter arterial chemoembolization., J. Control. Release. 50 (1998) 135-43. http://www.ncbi.nlm.nih.gov/pubmed/9685880 (accessed August 22, 2018).

[8] F. Deschamps, G. Farouil, W. Gonzalez, C. Robic, A. Paci, L.M. Mir, L. Tselikas, T. de Baère, Stabilization Improves Theranostic Properties of Lipiodol@-Based Emulsion During Liver Trans-arterial Chemo-embolization in a VX2 Rabbit Model, Cardiovasc. Intervent. Radiol. 40 (2017) 907-913. 
doi:10.1007/s00270-017-1616-2.

[9] N. Ménard, N. Tsapis, C. Poirier, T. Arnauld, L. Moine, C. Gignoux, F. Lefoulon, J.-M. Péan, E. Fattal, Novel Surfactants with Diglutamic Acid Polar Head Group: Drug Solubilization and Toxicity Studies, Pharm. Res. 29 (2012) 1882-1896. doi:10.1007/s11095-012-0714-8.

[10] N. Branco, I. Lee, H. Zhai, H.I. Maibach, Long-term repetitive sodium lauryl sulfate-induced irritation of the skin: an in vivo study, Contact Dermatitis. 53 (2005) 278-284. doi:10.1111/j.01051873.2005.00703.x.

[11] R. Liu, ed., Water-Insoluble Drug Formulation, Second Edition, CRC Press, 2008. doi:10.1201/9781420009552.

[12] D. Attwood, A.T. Florence, Aspects of surfactant toxicity, in: Surfactant Syst., Springer Netherlands, Dordrecht, 1983: pp. 614-697. doi:10.1007/978-94-009-5775-6_10.

[13] W. Ramsden, Separation of Solids in the Surface-Layers of Solutions and "Suspensions" (Observations on Surface-Membranes, Bubbles, Emulsions, and Mechanical Coagulation). -- Preliminary Account, Proc. R. Soc. London. 72 (1903) 156-164. doi:10.1098/rspl.1903.0034.

[14] S.U. Pickering, CXCVI.-Emulsions, J. Chem. Soc., Trans. 91 (1907) 2001-2021. doi:10.1039/CT9079102001.

[15] Y. Chevalier, M.A. Bolzinger, Emulsions stabilized with solid nanoparticles: Pickering emulsions, Colloids Surfaces A Physicochem. Eng. Asp. 439 (2013) 23-34. doi:10.1016/j.colsurfa.2013.02.054.

[16] C.P. Whitby, L.H. Lim, N. Ghouchi Eskandar, S. Simovic, C.A. Prestidge, Poly(lactic-co-glycolic acid) as a particulate emulsifier, J. Colloid Interface Sci. 375 (2012) 142-147. doi:10.1016/j.jcis.2012.02.058.

[17] L.A. Guzman, V. Labhasetwar, C. Song, Y. Jang, A.M. Lincoff, R. Levy, E.J. Topol, Local intraluminal infusion of biodegradable polymeric nanoparticles. A novel approach for prolonged drug delivery after balloon angioplasty., Circulation. 94 (1996) 1441-8. http://www.ncbi.nlm.nih.gov/pubmed/8823004 (accessed August 22, 2018).

[18] N. Grabowski, H. Hillaireau, J. Vergnaud, N. Tsapis, M. Pallardy, S. Kerdine-Römer, E. Fattal, Surface coating mediates the toxicity of polymeric nanoparticles towards human-like macrophages., Int. J. Pharm. 482 (2015) 75-83. doi:10.1016/j.ijpharm.2014.11.042.

[19] N. Grabowski, H. Hillaireau, J. Vergnaud-Gauduchon, V. Nicolas, N. Tsapis, S. Kerdine-Römer, E. Fattal, Surface-Modified Biodegradable Nanoparticles' Impact on Cytotoxicity and Inflammation Response on a Co-Culture of Lung Epithelial Cells and Human-Like Macrophages., J. Biomed. Nanotechnol. 12 (2016) 135-46. http://www.ncbi.nlm.nih.gov/pubmed/27301179 (accessed August 27, 2018).

[20] T. de Baere, Y. Arai, R. Lencioni, J.-F. Geschwind, W. Rilling, R. Salem, O. Matsui, M.C. Soulen, Treatment of Liver Tumors with Lipiodol TACE: Technical Recommendations from Experts Opinion, Cardiovasc. Intervent. Radiol. 39 (2016) 334-343. doi:10.1007/s00270-015-1208-y. 
[21] L.N. Okassa, H. Marchais, L. Douziech-Eyrolles, K. Hervé, S. Cohen-Jonathan, E. Munnier, M. Soucé, C. Linassier, P. Dubois, I. Chourpa, Optimization of iron oxide nanoparticles encapsulation within poly(d,1-lactide-co-glycolide) sub-micron particles, Eur. J. Pharm. Biopharm. 67 (2007) 31-38. doi:10.1016/j.ejpb.2006.12.020.

[22] F. Deschamps, L. Moine, T. Isoardo, L. Tselikas, A. Paci, L.M. Mir, N. Huang, E. Fattal, T. de Baère, Parameters for Stable Water-in-Oil Lipiodol Emulsion for Liver Trans-Arterial Chemo-Eembolization., Cardiovasc. Intervent. Radiol. 40 (2017) 1927-1932. doi:10.1007/s00270-017-1763-5.

[23] M. Destribats, S. Gineste, E. Laurichesse, H. Tanner, F. Leal-Calderon, V. Héroguez, V. Schmitt, Pickering emulsions: what are the main parameters determining the emulsion type and interfacial properties?, Langmuir. 30 (2014) 9313-26. doi:10.1021/la501299u.

[24] I. Bala, S. Hariharan, M.N.V.R. Kumar, PLGA nanoparticles in drug delivery: the state of the art., Crit. Rev. Ther. Drug Carrier Syst. 21 (2004) 387-422. http://www.ncbi.nlm.nih.gov/pubmed/15719481 (accessed August 22, 2018).

[25] B.P. Binks, ed., Modern Aspects of Emulsion Science, Royal Society of Chemistry, Cambridge, 1998. doi:10.1039/9781847551474.

[26] T. de Baere, J. Dufaux, A. Roche, J.L. Counnord, M.F. Berthault, A. Denys, P. Pappas, Circulatory alterations induced by intra-arterial injection of iodized oil and emulsions of iodized oil and doxorubicin: experimental study., Radiology. 194 (1995) 165-70. doi:10.1148/radiology.194.1.7997545.

[27] T.F. Tadros, Emulsion Formation, Stability, and Rheology, (2013).

[28] D. Quemada, Rheology of concentrated disperse systems and minimum energy dissipation principle, Rheol. Acta. 16 (1977) 82-94. doi:10.1007/BF01516932.

[29] B.P. Binks, P.D.I. Fletcher, B.L. Holt, P. Beaussoubre, K. Wong, Phase inversion of particle-stabilised perfume oil-water emulsions: Experiment and theory, Phys. Chem. Chem. Phys. 12 (2010) 1195411966. doi:10.1039/c0cp00558d.

[30] B.P. Binks, J.H. Clint, Solid wettability from surface energy components: Relevance to pickering emulsions, Langmuir. 18 (2002) 1270-1273. doi:10.1021/la011420k.

[31] I. Okayasu, S. Hatakeyama, T. Yoshida, S. Yoshimatsu, K. Tsuruta, H. Miyamoto, Y. Kimula, Selective and persistent deposition and gradual drainage of iodized oil, Lipiodol in the hepatocellular carcinoma after injection into the feeding hepatic artery., Am. J. Clin. Pathol. 90 (1988) 536-44. http://www.ncbi.nlm.nih.gov/pubmed/2459957 (accessed August 22, 2018).

[32] F. Laredj-Bourezg, Y. Chevalier, O. Boyron, M.-A. Bolzinger, Emulsions stabilized with organic solid particles, Colloids Surfaces A Physicochem. Eng. Asp. $413 \quad$ (2012) 252-259. doi:10.1016/J.COLSURFA.2011.12.064.

[33] F. Laredj-Bourezg, M.-A. Bolzinger, J. Pelletier, M.-R. Rovere, B. Smatti, Y. Chevalier, Pickering Emulsions Stabilised by Biodegradable Particles Offer a Double Level of Controlled Delivery of 
Hydrophobic Drugs, in: 2013: pp. 143-156. doi:10.1039/9781849734639-00143.

[34] F. Qi, J. Wu, G. Sun, F. Nan, T. Ngai, G. Ma, Systematic studies of Pickering emulsions stabilized by uniform-sized PLGA particles: preparation and stabilization mechanism, J. Mater. Chem. B. 2 (2014) 7605-7611. doi:10.1039/C4TB01165A.

[35] S. Arditty, C.P. Whitby, B.P. Binks, V. Schmitt, F. Leal-Calderon, Some general features of limited coalescence in solid-stabilized emulsions., Eur. Phys. J. E. Soft Matter. 11 (2003) $273-281$. doi:10.1140/epje/i2003-10018-6.

[36] E. Lilienberg, I.R. Dubbelboer, A. Karalli, R. Axelsson, T.B. Brismar, C.E. Barbier, A. Norén, F. Duraj, M. Hedeland, U. Bondesson, E. Sjögren, P. Stal, R. Nyman, H. Lennernäs, In vivo drug delivery performance of lipiodol-based emulsion or drug-eluting beads in patients with hepatocellular carcinoma, Mol. Pharm. 14 (2017) 448-458. doi:10.1021/acs.molpharmaceut.6b00886. 